\title{
Electron Microscopic Study of Adrenal Cortex in Spontaneously Hypertensive Rat
}

\author{
Kioko Kawai, M.D., Hideo Tsuchiyama, M.D., \\ and Hajime Sugihara, M.D.
}

It has been known that adrenal cortex plays a significant role in the pathogenesis and maintenance of hypertension. We have shown some characteristic ultrastructural alterations in the adrenal cortex of spontaneously hypertensive rats in various stages of hypertension. We have also discussed them in relation to the functional state of adrenals. However it is still not determined the critical stage of the occurrence of these changes.

In this study, the adrenal cortices of the fetal rats in SHR, 19 and 21 days of gestation, were examined by electron microscope. And we compared the appearances of the fine structure in fetal rats with those in prehypertensive, initial and advanced stage of hypertension.

In the prehypertensive rats, the Golgi complex of the cells in the zona glomerulosa was moderately enlarged and there were a few elongated large mitochondria which had cristae with shelf-like parallel array of tubules. At this stage, the cell organelles of the fascicular zone in SHR did not differ from control rats.

In the zona glomerulosa cells at the initial stage of hypertension, Golgi complex with dilated cisternae and dense bodies were well developed and the latter were scattered in and around the Golgi complex. Similar to the prehypertensive rats, elongated mitochondria containing shelf-like parallel array of tubules were often observed. These findings were more prominent in the zona glomerulosa cells at the initial stage of hypertension than that of the prehypertensive stage.

In the zona fasciculata of this stage, smooth surfaced endoplasmic reticulum was increased and dilated. Well developed mitochondria containing vesicular cristae was also noted.

The alterations of zonal fine structure in the advanced stage of hypertension were similar to those observed in the initial stage of hypertension. In addition to these findings, increased number of dense bodies and lysosomes including lipofuscin pigment were observed in the fascicular zone. In the advanced stage of hypertension, we often encountered the nodular portions in the fascicular and reticular zone of SHR. The characteristic electron

From the Department of Pathology, Faculty of Medicine, Nagasaki University, Nagasaki. 
microscopic findings of the cells in these nodules were numerous mitochondria with lamellar circular cristae, intramitochondrial granules and lamellar arrangement of smooth surfaced endoplasmic reticulum around lipid droplets.

In 21 days of gestation in SHR, the cells of the subcapsular area in the adrenal cortex were somewhat elongated and had a large irregular shaped nucleus with prominent nucleolus. Oval or elongated mitochondria with lamellar or tubular cristae was sparse. But shelf-like parallel array of tubules was not observed in these mitochondria. Golgi complex was not well developed and lipid vacuoles were scarcely seen.

The cells of zona fasciculata had a broad cytoplasm. Mitochondria was round in shape and contained vesicular cristae. It was difficult to find the remarkable differences in the cortical fine structure between SHR and control rats.

On the basis of these findings, it may be concluded that hyperactive state of mineralocorticoid secretion is already present in the prehypertensive stage of SHR and it becomes more prominent in the initial stage of hypertension. Furthermore, the hyperfunctional state of the fascicular zone may also occur during the development of hypertension. The adrenal cortex of SHR in the advanced stage of hypertension still shows hyperactive changes in the either zones, but they tend to approach a state of exhaustion on the other hand. In the adrenal cortex of fetal stage, there were no characteristic changes resembling those of various stages in SHR after birth. 